\title{
Effects of prior exploration on subsequent discrimination learning
}

\author{
Mitri E. Shanab / \\ UNIVERSITY OF CALIFORNIA, SANTA BARBARA
}

\begin{abstract}
Abstraet
Two groups of rats learned a positional discrimination task on an elevated "Y"maze. Prior to discrimination training the experimental group was allowed to explore the maze for nine days during which time the control group was given pretraining on an elevated straightaway. It was found that the experimental group learned the discrimination task significantly faster than the control group. This superiority was interpreted as due to the fact that the experimental group started discrimination learning with an already formed habit strength $(\mathrm{H})$, which by interacting with incentive motivation $(\mathrm{K})$ in a multiplicative way produced a larger excitatory potential (E) than the control group which started discrimination learning with negligible habit strength.

\section{Problem}

The question of how much previous learning influences later learning continues to plague students of learning in general and of transfer of training in particular. The hotly debated but now dormant controversy over latent learning is a case in point. The present study was carried out in an effort to provide a more precise interpretation of the behavior of latent learning animals during exploration.

Oakes (1956) had 24 rats explore a three-platform elevated "Y" maze for 12 days, after which they were fed on a particular platform for three days and then tested to see if they could go directly to their feeding platform when placed on a different platform. Of the remaining 21 rats, 16 made the correct choice $(p<.05)$, from which Oakes concluded that the rats 'had learned something about the layout of the apparatus during the familiarization phase when no obvious reinforcers were present in the situation." It was felt that such a conclusion was not warranted by the data since the study did not include a control group receiving no prior exploration experience. When the proper control group was included by the writer in an unpublished replication of Oakes' study, both the exploration and the nonexploration groups performed at chance level during the critical test. However, the group with prior exploration ran significantly faster than the control group, suggesting the hypothesis that prior exploration of a particular maze may have a differential effect upon the development of the motor skill of running on that maze rather than the cognitive map of the maze. If this is the case, then a group which has explored a maze for some time should later learn a discrimination task in that maze faster than a group which has had no such exploration experience. The present study tests this inference.

\section{Method}

The Ss, 30 male albino rats of the Houston Cheeks strain and approximately 70 days old, were divided randomly into two equal-sized experimental and control groups.

As in the Oakes study the apparatus used was a 4-ft open elevated "Y" maze consisting of three 6-ft runways each terminating in a $12 \times 14$ in platform. The platforms each had a different surface covering: a metal washboard surface, a glass surface, and a coarse sandpaper surface.

All Ss were housed in individual cages. Each S was handled $5 \mathrm{~min}$. a day for seven days while under a 23- hr. food deprivation. The experimental Ss were then allowed to explore the maze $10 \mathrm{~min}$. a day for nine days, during which time no food was placed in any part of the maze. During the exploration phase each $\mathrm{S}$ was placed on one platform an equal number of times in accordance with a prearranged random schedule. The control Ss during this time were given pretraining trials on a 3-ft elevated straightaway. Following exploration both groups were trained on a positional discrimination task. To control against natural preferences for any one platform, each group was divided into three equal subgroups which were trained to run to different platforms. Similarly, to control for turning preferences, each $\mathrm{S}$ in the subgroup was randomly assigned to one platform, so that for roughly half the Ss a correct response was a right turn, while for the rest a left turn led to the correct platform.

Discrimination training consisted of eight trials a day with $15 \mathrm{sec}$. as the minimum intertrial interval. Each correct response was rewarded with a $45 \mathrm{mg}$ pellet. All Ss were trained to a criterion of seven consecutively correct responses out of eight responses spanning two training sessions.

\section{Results}

The mean number of trials to criterion for the experimental group and the control group were 8.9 and 23.9 respectively. Because of the marked skewness of the distribution of scores a Wilcoxon two-sample non-parametric rank test was done which revealed a definite superiority of the experimental group over the control group $(p=.0002)$. In addition, the data were subjected to a two-way analysis of variance test, in which correct platforms and groups formed the main variables. A highly significant group effect $(F=16.82$; $\mathrm{df}=1,24 ; \mathrm{p}<.001$ ) appeared, but the $\mathrm{F}$ for platforms was less than unity and clearly nonsignificant. There was no interaction effect between group and platform $(F=2.47$; 
$\mathrm{df}=2,24 ; \mathrm{p}<.10$ ), indicating that the superiority of the experimental group over the control group was not dependent upon the particular platform to which the Ss were trained.

\section{Discussion}

The results support the hypothesis proposed in this study, namely, that a group receiving prior exploration on an elevated maze would learn a subsequent discrimination task faster than a group receiving no prior exploration experience. The findings are consistent with Spence's (1956) formulations, which assume that habit strength builds up as the result of the S's making instrumental responses to stimuli in the experimental situation, regardless of whether or not these responses are rewarded. In the absence of any incentive motivation $(\mathrm{K})$, and with drive held constant, habit strength $(\mathrm{H})$ will not show up in reaction potential $(\mathrm{E})$, as can be shown by the following simple equation:
$\mathrm{E}=\mathrm{cHK}$, where $\mathrm{c}$ stands for constant drive.

It is clear that as long as $\mathrm{K}$ is near zero, as is the case during nonrewarded exploration, the $H$ which builds up during this time will not be reflected in perfor mance. However, with the introduction of food during discrimination learning, $\mathrm{K}$ starts to build up through the $\mathrm{r}_{\mathrm{g}}-\mathrm{s}_{\mathrm{g}}$ mechanism (Spence, 1956), and to interact with the already existing habit strength. This interaction would soon be reflected in the S's discrimination behavior, and hence account for the superior performance of the exploring rat over the nonexploring rat.

\section{References}

OAKES, W. F. Latent learning in the three-table apparatus. J. exp. Psychol., 1956, 51, 287-289.

SPENCE, K. W. Behavior theory and conditioning. New Haven: Yale University Press, 1956.

Note

1. Thanks are due to Dr. John W. Cotton for his valuable advice. 